\title{
Engineered Adenosine-Releasing Cells for Epilepsy Therapy: Human Mesenchymal Stem Cells and Human Embryonic Stem Cells
}

\author{
Detlev Boison \\ R. S. Dow Neurobiology Laboratories, Legacy Research, Portland Oregon 97232
}

\begin{abstract}
Summary: Adenosine is a modulator of neuronal activity with anticonvulsant and neuroprotective properties. Conversely, focal deficiency in adenosine contributes to ictogenesis. Thus, focal reconstitution of adenosine within an epileptogenic brain region constitutes a rational therapeutic approach, whereas systemic augmentation of adenosine is precluded by side effects. To meet the therapeutic goal of focal adenosine augmentation, genetic disruption of the adenosine metabolizing enzyme, adenosine kinase (ADK) in rodent cells was used as a molecular strategy to induce adenosine release from cellular brain implants, which demonstrated antiepileptic and neuroprotective properties. Currently, the second generation of adenosine-releasing cells is under development based on the rationale to use human stem cell-derived brain implants to avoid xenotransplantation. To effectively engineer human stem cells to release adenosine, a lentiviral vector was constructed to express inhibitory micro-RNA directed against
\end{abstract}

ADK. Lentiviral knockdown of ADK induced therapeutic adenosine release in human mesenchymal stem cells, which reduced acute injury and seizures, as well as chronic seizures, when grafted into the mouse hippocampus. The therapeutic potential of this approach suggests the feasibility to engineer autologous adenosine-releasing stem cells derived from a patient. Human embryonic stem cells (hESCs) have a high proliferative capacity and can be subjected to specific cellular differentiation pathways. hESCs, differentiated in vitro into neuroepithelial cells and grafted into the mouse brain, displayed intrahippocampal location and neuronal morphology. Using the same lentiviral micro-RNA vector, we demonstrated knockdown of ADK in hESCs. New developments and therapeutic challenges in using human mesenchymal stem cells and hESCs for epilepsy therapy will be critically evaluated. Key Words: Adenosine, epilepsy, cell therapy, stem cells, RNAi, rodent models.

\section{INTRODUCTION}

Despite the advent of a new armamentarium of antiepileptic drugs in recent years, about one third of all patients with epilepsy remains refractory to treatment, and widespread systemic and central side effects limit the most optimal use of these drugs. ${ }^{1}$ In contrast, focal drug delivery to epileptogenic brain regions is considered to be a promising and safe alternative to limit side effects of conventional pharmacotherapy. ${ }^{2}$ Based on these considerations, cell therapies have been evaluated for the treatment of epilepsy, not only for cell-based drug delivery, but also in attempts to reconstruct or repair damaged circuitry in epilepsy. ${ }^{3-6}$ The goals for cellbased epilepsy therapy are twofold. On one hand thera-

Address correspondence and reprint requests to: Detlev Boison, Ph.D., Robert Stone Dow Neurobiology Laboratories, Legacy Research, 1225 N.E. 2nd Avenue, Portland, OR 97232. E-mail: dboison@downeurobiology.org. peutic cell implants can be used to replace neurons lost to epilepsy-associated cell death and to reconstruct damaged hippocampal circuitry, as has been demonstrated in an elegant series of experiments by Shetty and colleagues. ${ }^{7-10}$ These approaches have recently been reviewed $^{5}$ and require differentiation of implanted cells into specific types of neurons and, finally, functional integration of these cells into pre-existing neuronal networks. After intrahippocampal grafting of fetal hippocampal neurons, remarkable therapeutic outcomes were achieved suggesting reconstitution of the disrupted circuitry. ${ }^{7}$ On the other hand, cellular transplants can be used as vehicles for the delivery of endogenous neurotrophic compounds, or when genetically engineered, for the delivery of anticonvulsants, such as adenosine, ${ }^{11}$ or gamma-aminobutyric acid (GABA). ${ }^{12}$ In these approaches paracrine drug delivery is usually sufficient and functional differentiation and integration of the cells is not necessary, although beneficial synergistic effects between paracrine drug delivery and endogenous stem cell-dependent trophic 
effects have been described. ${ }^{13,14}$ Potential sources for therapeutic cells are fetal cells (e.g., noradrenergic, cholinergic, GABAergic), neuronal stem cells, embryonic or adult stem cells, or cells genetically engineered to release therapeutic compounds. The challenges that need to be met are manifold and require long-term survival and effectiveness after grafting, immunocompatibility, and efficacy in pharmacoresistant epilepsy. Several approaches require neuronal differentiation, functional integration, and network interactions.

\section{THE RATIONALE FOR ADENOSINE-BASED CELL THERAPIES}

The role of the purine ribonucleoside adenosine as an endogenous regulator of hippocampal activity has first been described more than 25 years ago, ${ }^{15}$ and the potent anti-ictogenic ${ }^{14,16-21}$ and neuroprotective $e^{22-24}$ properties of adenosine are well documented. Adenosine controls neuronal activity by activation of pre- and postsynaptic adenosine receptors $\left(\mathrm{A}_{1}, \mathrm{~A}_{2 \mathrm{~A}}, \mathrm{~A}_{2 \mathrm{~B}}\right.$, and $\left.\mathrm{A}_{3} \mathrm{R}\right)$ that are coupled to inhibitory $\left(A_{1}\right.$ and $\left.A_{3}\right)$ or stimulatory $\left(A_{2 A}\right.$ and $A_{2 B}$ ) G-proteins. ${ }^{25-28}$ Whereas $A_{1}$ Rs mediate tonic heterosynaptic depression largely by inhibition of glutamate release and stabilization of the postsynaptic membrane potential, $\mathrm{A}_{2 \mathrm{~A}} \mathrm{Rs}$ may potentiate high-frequency stimulations within a globally inhibited network. ${ }^{29}$ Consequently, $A_{1}$ Rs are ideally suited to prevent the spread of hyperexcitability, a hypothesis that has been confirmed. ${ }^{30,31}$ Synaptic levels of adenosine are largely regulated by an astrocyte-driven adenosine cycle ${ }^{32}$ and the activity of the astrocyte-based adenosine-removing enzyme adenosine kinase (ADK). ${ }^{18}$ A recent study from our laboratory has identified the enzyme ADK in astrocytes as a molecular link between astrogliosis and neuronal dysfunction in epilepsy. ${ }^{21}$ In a mouse model of CA3-selective epileptogenesis, we demonstrated acute injury, subsequent astrogliosis with concomitant upregulation of ADK (leading to a local adenosine deficit), and spontaneous electrographic seizures, all restricted to the CA3 ipsilateral to a preceding intra-amygdaloid injection of the excitotoxin kainic acid (KA). Likewise, transgenic overexpression of ADK triggered spontaneous seizures, whereas transgenic mice with a forebrainselective reduction of ADK were resistant to seizure development. ${ }^{21}$ These studies suggest that glial dysfunction contributes to epilepsy. This includes adenosine deficiency linked to astrogliosis, a pathological hallmark of the epileptic brain.

The "ADK hypothesis of epileptogenesis"17 implies that any type of brain injury can trigger astrogliosis, possibly via an injury-related, acute surge of micromolar levels of adenosine. Astrogliosis in turn leads to upregulation of $\mathrm{ADK}$, creating focal adenosine deficiency as direct cause for seizures. Therefore, adenosine augmen- tation therapies (AATs) ${ }^{17}$ constitute a rational approach for therapeutic intervention, substantiated by findings that systemic AATs are effective in preventing spontaneous seizures in mice that are resistant to conventional antiepileptic drugs. ${ }^{33,34}$ Systemic AAT, unfortunately, is not a therapeutic option due to widespread peripheral and central side effects. $A_{1} R$ agonists, when given systemically have profound peripheral, mainly cardiovascular, effects. ${ }^{35}$ Likewise, the use of systemic ADK inhibitors is restricted due to liver toxicity of ADK deficiency ${ }^{36}$ and the incidence of brain hemorrhage in rats and dogs after application of the ADK inhibitor GP-3966. ${ }^{37}$

\section{ADENOSINE-RELEASING STEM CELLS FOR EPILEPSY THERAPY}

As previously outlined, AATs constitute a neurochemical rationale for the suppression or prevention of seizures in epilepsy. To circumvent side effects of systemic AATs, focal AATs were tested by transplantation of adenosine-releasing cells into the vicinity of an epileptogenic focus. ${ }^{11}$ The first generation of therapeutic implants was based on rodent fibroblasts engineered to release adenosine. These cells were encapsulated into semi-permeable polymer membranes to prevent immune-rejection and to prevent network interactions. Intraventricular implants of these devices provided nearly complete protection from seizures in kindled rats, which was limited to 2 to 4 weeks due to poor, long-term survival of the encapsulated cells. ${ }^{38}$ These results also demonstrated that local paracrine release of adenosine is sufficient to prevent seizures; thus, functional integration of therapeutic cells into hippocampal networks is not necessary.

The second generation of adenosine-releasing therapeutic cells was generated in our laboratory by bi-allelic genetic disruption of the $A d k$-gene in mouse embryonic stem cells. ${ }^{39}$ The cells were subjected to a neural differentiation protocol ${ }^{40}$ in vitro. Resulting neural precursor cells (NPs) released adenosine and were transplanted into the infrahippocampal fissure of rats prior to hippocampal kindling. When analyzed 26 days after grafting, we found dense clusters of graft-derived cells within the infrahippocampal fissure that likely formed a reservoir for the paracrine release of adenosine. In addition, graft-derived cells migrated into the ipsilateral CA1, stained positive for NeuN, and assumed a neuronal morphology with long, branching processes. ${ }^{14}$ These data demonstrate that adenosine-releasing, stem cell derived, brain implants display improved survival characteristics compared with encapsulated cell grafts. One week after grafting kindling was initiated, the subsequent increase in seizure activity was compared with recipients of corresponding wild-type (WT) cells and sham-operated animals. Strikingly, kindling in recipients of adenosine- 
releasing, embryonic stem-derived, NPs was strongly retarded. ${ }^{14}$ Thus, 22 days after grafting, and after 48 kindling stimulations, recipients of adenosine-releasing NPs failed to display generalized (stages 4 and 5) seizures; instead, these animals displayed more immature kindling parameters. This delay in the progressive development of behavioral seizures was observed in the presence of electrographic afterdischarges elicited by each kindling stimulation. These findings suggest a novel antiepileptogenic or disease-modifying function of mediated stem cell adenosine delivery; using this approach, however, true antiepileptogenic effects are difficult to assess due to overlapping anti-ictogenic effects of adenosine. In addition, this study left open the question as to whether ADK-deficient, stem cell derived, brain implants would be equally effective in epileptogenesis models that involve astrogliosis and spontaneous recurrent seizures.

To assess potential antiepileptogenic effects of adenosine-releasing, mouse embryonic stem cell derived, NPs in a model that involves astrogliosis and the development of spontaneous seizures, we chose a mouse model of CA3-selective epileptogenesis. ${ }^{21}$ Twenty-four hours after unilateral intra-amygdaloid injection of KA (initial epileptogenesis-precipitating injury) ADK-deficient NPs were injected into the infrahippocampal fissure ipsilateral to the KA injection. Controls received respective WT cells or a corresponding sham procedure. When analyzed 3 weeks later, all graft recipients had dense clusters of graft-derived cells located within the infrahippocampal fissure. In addition, individual cells had migrated into the ipsilateral CA1 and assumed a neuronal morphology. In all animals, the CA3-selective initial epileptogenesis-precipitating injury was confirmed by histological analysis. Most importantly, recipients of adenosine-releasing $A d k^{-1}$ NPs were characterized by a significant reduction in astrogliosis and by almost normal ADK levels in the ipsilateral CA3, whereas prominent astrogliosis and upregulation of ADK was found in the CA3 of control animals. These findings indicate that in the recipients of adenosine-releasing, stem cell derived, brain implants, two important features of epileptogenesis (i.e., astrogliosis and upregulation of ADK) were significantly reduced. In concordance with normal ADK levels, all recipients of adenosine-releasing cells were completely protected from any seizure activity, whereas respective control animals displayed $>4$ electrographic seizures per $h$. Thus, adenosine-releasing, stem cell-derived, brain implants prevented the expression of seizures in a spontaneous seizure model. ${ }^{21}$ Reduced astrogliosis and lack of ADK-upregulation in the therapeutic group suggest that adenosine-releasing, stem cell derived, brain implants exert at least some antiepileptogenic effects. Indeed, adenosine acting on astrocytic adenosine $A_{1}$ receptors was shown to inhibit reactive astrogliosis. ${ }^{41}$ Reduced astrogliosis in turn would limit epileptogenic upregulation of $\mathrm{ADK}$, thus ameliorating the seizure-triggering adenosine deficiency.

\section{HUMAN ADENOSINE-RELEASING STEM CELLS FOR EPILEPSY THERAPY?}

The promises and potential pitfalls of human embryonic stem cell (hESC) therapy for brain repair have recently been reviewed. ${ }^{42,43}$ hESCs are usually derived from the inner cell mass of human 4.5-day-old pre-implantation embryos obtained after in vitro fertilization. These cells have an unlimited capacity for self-renewal in culture and under the right conditions they are capable to differentiate into any adult cell type. Although hESCs are believed to offer potential cures and therapies for many devastating diseases, ${ }^{42}$ research using them is still in its early stages since their discovery in $1998^{44}$ and initiation of federal funding on August 9, 2001. Apart from ethical concerns, several scientific issues need to be resolved before hESCs can safely be used in human patients: ${ }^{42}$ 1) For optimized functional outcomes, differentiation needs to be controlled by culture conditions, genetic modification, or selection procedures; 2) for safety reasons, tumor formation needs to be excluded by enrichment of nontumorigenic cells or depletion of tumorigenic cells; 3 ) safety reasons also require the use of early passages and karyotyping to exclude genetic aberrations; and 4) for optimized graft survival, inflammation and graft rejection needs to be prevented by immunosuppression, the induction of immunotolerance, or somatic cell nuclear transfer. Several protocols have been developed to direct hESCs into neuronal differentiation pathways in vitro. ${ }^{45-48}$ Accordingly, hESCs can be differentiated under controlled conditions into NPs by two major steps: ${ }^{47}$ 1) growth of clusters of hESCs in chemically defined serum-free suspension culture, followed by 2) controlled and efficient differentiation into NPs by growing the cells in the presence of the growth and differentiation-inducing factors noggin and basic fibroblast growth factor (bFGF). hESC-derived NPs were shown to functionally engraft into rodent brain and to improve behavioral and functional deficits in Parkinsonian rats. ${ }^{49-51}$ Despite this recent progress many critical issues remain. ${ }^{42}$

To investigate whether hESCs might be of use in animal models of epilepsy, we tested whether hESCderived NPs can integrate and survive in the epileptic mouse brain. According to established protocols ${ }^{52}$ hESCs were differentiated into neuroepithelial cells and labeled with a fluorescent tracer. hESC-derived neuroepithelial cells were grafted into the infrahippocampal fissure of mice $24 \mathrm{~h}$ after the intra-amygdaloid injection of KA. Three weeks later, all animals $(n=6)$ had developed spontaneous seizures. Histological analysis revealed dense clusters of graft-derived cells within the 

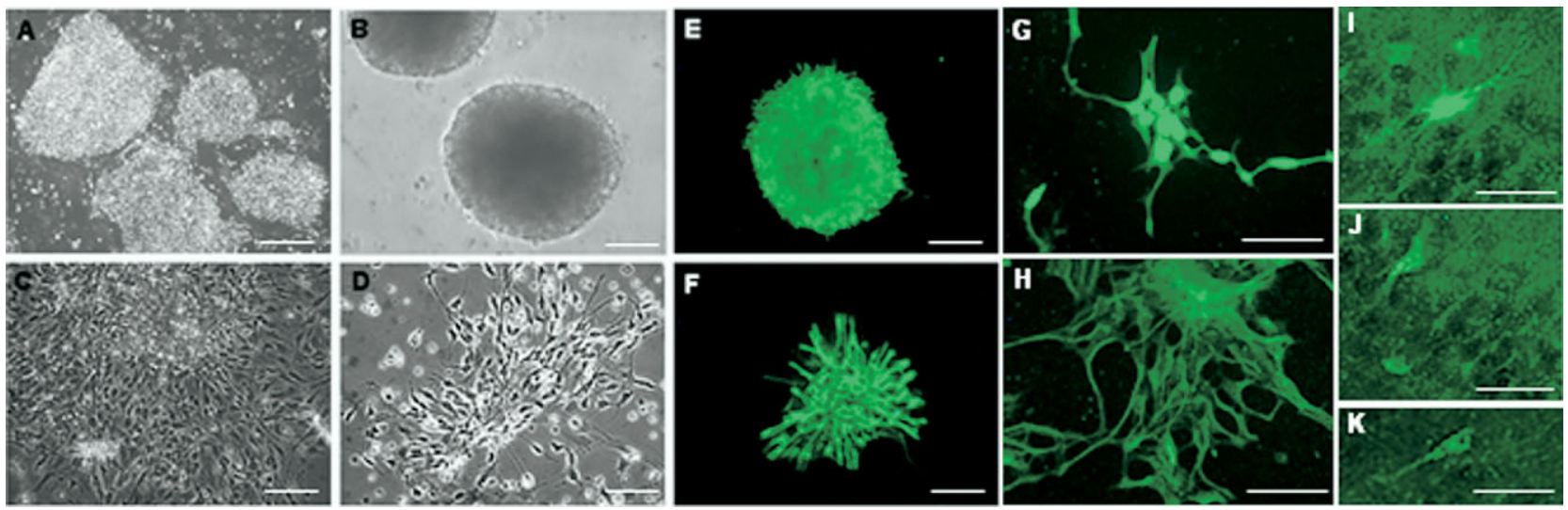

FIG. 1. Different stages of human embryonic stem cells differentiation (hESCSs). A: Undifferentiated hESCs. B: hESC-derived neurosphere. C: Neurosphere after attachment. D: hESC-derived neural precursor cells. E: Nestin-stained neurosphere. F: Nestin-stained neural precursor (NP) cells. G: Pax-1 positive cells after neuronal differentiation. H: tau-1 positive cells after neuronal differentiation. I, J, and K: Human NP-derived cells with neuronal morphology located within the CA1 region of an epileptic mouse 3 weeks after transplantation. Scale: A-F: $250 \mathrm{~mm}$; G, H: $100 \mathrm{~mm}$; and I-K: $25 \mathrm{~mm}$. This figure summarizes unpublished data from the Boison lab.

infrahippocampal fissure and individual graft-derived cells that had migrated and integrated into the ipsilateral CA1 (FIG. 1). These WT cells had no influence on the development of spontaneous seizures in the host animals. Thus, an efficient method is needed to engineer human stem cells for therapeutic adenosine delivery before hESCs can be considered for epilepsy therapy.

\section{HUMAN MESENCHYMAL STEM CELLS FOR EPILEPSY THERAPY?}

Human mesenchymal stem cells (hMSCs) derived from bone marrow constitute an easily accessible cell source and therefore have potential for autologous grafting. So far, prior clinical experience with hMSC transplantation is largely limited to bone formation. ${ }^{53}$ In rodents, hMSC transplantation led to remarkable results in models of stroke $\mathrm{e}^{5,55}$ and traumatic brain injury. ${ }^{56}$ In contrast to the use of hESCs, no ethical concerns limit the clinical use of hMSCs. The hMSCs engineered to release adenosine might be considered for autologous cell therapy for epilepsy. Several issues need to be considered: 1) There is little prior experience with hMSCs in epilepsy models; 2) hMSC-implants need to survive for a sufficiently long period of time; and 3) it needs to be clarified whether specific differentiation of the implanted cells is needed or whether paracrine release of adenosine is sufficient for therapeutic effects. In order to develop hMSCs for therapeutic adenosine release we recently developed a lentiviral vector that expresses an inhibitory micro-RNA (miRNA) directed against ADK.$^{57}$ hMSCs transduced with this vector were characterized by up to $80 \%$ downregulation of ADK. After cultivating $10^{5}$ ADK-knockdown cells for $8 \mathrm{~h}$, a concentration of $8.5 \mathrm{ng}$ adenosine per $\mathrm{mL}$ of medium was found, whereas control of hMSCs failed to release adenosine. ADK-knockdown hMSCs and control cells transduced with a scrambled control sequence, along with a sham control, were transplanted into the infrahippocampal cleft of mice 1 week before the intraamygdaloid injection of KA. All graft recipients had dense hMSC-derived cell clusters spreading throughout the ipsilateral infrahippocampal fissure when analyzed 8 days after implantation. To assess the therapeutic effects of the grafts, animals were analyzed for acute KA-induced seizures and the extent of seizureinduced neuronal cell loss. While control animals were characterized by KA-induced status epilepticus and subsequent neuronal cell loss, animals with therapeutic ADK knockdown implants displayed a 35\% reduction in seizure duration and $65 \%$ reduction in neuronal cell loss, when analyzed $24 \mathrm{~h}$ after KA. This study demonstrates that hMSC-derived brain implants with a lentiviral knockdown of ADK provide potent anticonvulsant and neuroprotective effects in an acute seizure and cell-death model. However, in a clinical scenario preventive cell therapy is rather unlikely to be justified. Therefore, it needs to be ascertained that therapeutic cell implants are effective after the injury had occurred. To investigate the therapeutic potential of adenosine-releasing hMSCs on the development of epilepsy after injury, we followed an experimental strategy in which we first injected KA into the amygdala of mice to create an initial epileptogenesisprecipitating injury. Then $24 \mathrm{~h}$ later, hMSCs with a knockdown of ADK were grafted into the infrahippocampal fissure of KA-injured mice. Controls consisted of sham-grafted animals and recipients of WT stem cells. Three weeks after grafting, all control animals had developed CA3-selective seizures $(4.2 \pm 1.4$ seizures per hour [seizures/h] with an average duration of $17.2 \pm 5.1 \mathrm{~s} ; \mathrm{n}=$ 6) in analogy to our previous study. ${ }^{21}$ In contrast, recipients of adenosine-releasing hMSCs were characterized by a significant reduction of both seizure frequency $(2.7 \pm 1.1$ seizures $/ \mathrm{h} ; p<0.001)$ and duration $(9.4 \pm 4.1 \mathrm{~s} ; p<$ 
Table 1. Adenosine-Releasing Human Stem Cells for Epilepsy Therapy: Properties of Embryonic and Mesenchymal Stem Cells

\begin{tabular}{|c|c|c|}
\hline & Human Embryonic Stem Cells & Human Mesenchymal Stem Cells \\
\hline Cell growth & Unlimited proliferation & Limited proliferation \\
\hline Directed differentiation & Necessary to avoid tumor formation & Not necessary \\
\hline Implant survival & $>3$ weeks $*$ & $>8$ weeks $*$ \\
\hline $\begin{array}{l}\text { Implant location after injection of cells } \\
\text { into infrahippocampal fissure }\end{array}$ & $\begin{array}{l}\text { Dense cell cluster in infrahippocampal } \\
\text { fissure plus "integration" into CA1* }\end{array}$ & $\begin{array}{l}\text { Dense cell cluster restricted to } \\
\text { infrahippocampal fissure }\end{array}$ \\
\hline Therapeutic effects & $\begin{array}{l}\text { Seizure suppression (?) } \\
\text { Disease modification (?) } \\
\text { Network modulation (?) }\end{array}$ & $\begin{array}{l}\text { Paracrine adenosine release: seizure } \\
\text { reduction and neuroprotection }\end{array}$ \\
\hline Immunology & $\begin{array}{l}\text { Requirement for immunosuppression } \\
\text { or therapeutic cloning }\end{array}$ & $\begin{array}{l}\text { Potential for autologous grafting } \\
\text { approach }\end{array}$ \\
\hline Ethics & Controversial & No concerns \\
\hline
\end{tabular}

*Unpublished data from our laboratory.

0.001). Treatment of the protected animals with the adenosine $\mathrm{A}_{1} \mathrm{R}$ antagonist 8-cyclopentyl-1,3-dipropylxanthine transiently restored a normal seizure pattern $(4.3 \pm 1.5$ seizures/h with duration of $22 \pm 13 \mathrm{~s}$ ) indicating that seizure suppression was due to graft-derived adenosine. In accordance with our previous studies ${ }^{21,57}$ all animals had dense clusters of hMSC-derived cells that maintained lentiviral gene expression, as became evident by emerald green fluorescent protein-driven green fluorescence. The two experiments described here, and unpublished studies from our laboratory demonstrate that hMSCs can survive within the infrahippocampal fissure for at least 8 weeks and provide therapeutic benefit by paracrine release of adenosine in both acute and chronic seizure models.

\section{CONCLUSIONS AND OUTLOOK}

Human stem cells engineered to release adenosine appear to be a promising approach to provide therapeutic benefit in both acute seizures and injury, as well as in chronic epilepsy. It needs to be noted, though, that all therapeutic effects previously described were obtained in adult rodents, reflecting a target population of adult patients with refractory temporal lobe epilepsy. For different age groups (e.g., present in childhood epilepsy), alternative adenosine augmentation approaches might be useful, such as ketogenic diets; these diets are frequently effective in childhood epilepsies and were recently suggested to augment adenosine levels in the brain. ${ }^{58}$

hMSCs and hESCs both have their distinctive advantages and disadvantages as summarized in Table 1. Nevertheless, considerable challenges remain before adenosine-based stem cell therapies can be translated into clinical use. These challenges include the demonstration of long-term seizure control, which in turn depends on cell viability and long-term maintenance of miRNA expression in vivo. It needs to be determined which doses of adenosine are most suitable to provide robust seizure protection without the induction of side effects. On the other hand, three recent studies ${ }^{14,20,21}$ suggest an antiepileptogenic potential of adenosine-releasing brain implants, an exciting possibility that needs to be explored further.

Acknowledgments: The work of the author is supported by grants RO1NS058780-01, R21NS057475-01, and R21NS05753801 from the National Institute of Neurological Disorders and Stroke (NINDS), the Good Samaritan Hospital Foundation, the Epilepsy Research Foundation through the generous support of Arlene \& Arnold Goldstein Family Foundation, and Citizens United in Research against Epilepsy (CURE) in collaboration with the Department of Defense (DoD).

\section{REFERENCES}

1. Vajda FJE. Pharmacotherapy of epilepsy: New armamentarium, new issues. J Clin Neurosci 2007;14:813-823.

2. Nilsen KE, Cock HR. Focal treatment for refractory epilepsy: hope for the future? Brain Res Brain Res Rev 2004;44:141-153.

3. Boison D. Cell and gene therapies for refractory epilepsy. Curr Neuropharmacol 2007;5:115-125.

4. Loscher W, Gernert M, Heinemann U. Cell and gene therapies in epilepsy - promising avenues or blind alleys? Trends Neurosci 2008;31:62-73.

5. Shetty AK, Hattiangady B. Concise review: Prospects of stem cell therapy for temporal lobe epilepsy. Stem Cells 2007;25:2396-2407.

6. Raedt R, Van Dycke A, Vonck K, Boon P. Cell therapy in models for temporal lobe epilepsy. Seizure-Eur J Epilepsy 2007;16:565-578.

7. Shetty AK, Hattiangady B. Restoration of calbindin after fetal hippocampal CA3 cell grafting into the injured hippocampus in a rat model of temporal lobe epilepsy. Hippocampus 2007;17:943956.

8. Rao MS, Hattiangady B, Rai KS, Shetty AK. Strategies for promoting anti-seizure effects of hippocampal fetal cells grafted into the hippocampus of rats exhibiting chronic temporal lobe epilepsy. Neurobiol Dis 2007:27:117-132.

9. Rao MS, Hattiangady B, Shetty AK. Fetal hippocampal CA3 cell grafts enriched with FGF-2 and BDNF exhibit robust long-term survival and integration and suppress aberrant mossy fiber sprouting in the injured middle-aged hippocampus. Neurobiol Dis 2006; 21:276-290.

10. Shetty AK, Zaman V, Hattiangady B. Repair of the injured adult hippocampus through graft-mediated modulation of the plasticity of the dentate gyrus in a rat model of temporal lobe epilepsy. J Neurosci 2005;25:8391-8401.

11. Boison D. Adenosine-based cell therapy approaches for pharmacoresistant epilepsies. Neurodegener Dis 2007;4:28-33. 
12. Thompson KW. Genetically engineered cells with regulatable GABA production can affect afterdischarges and behavioral seizures after transplantation into the dentate gyrus. Neuroscience 2005;133:1029-1037.

13. Pignataro G, Studer FE, Wilz A, Simon RP, Boison D. Neuroprotection in ischemic mouse brain induced by stem cell-derived brain implants. J Cereb Blood Flow Metab 2007;27:919-927.

14. Li T, Steinbeck JA, Lusardi T, Koch P, et al. Suppression of kindling epileptogenesis by adenosine releasing stem cell-derived brain implants. Brain 2007;130:1276-1288.

15. Dunwiddie TV. Endogenously released adenosine regulates excitability in the in vitro hippocampus. Epilepsia 1980;21:541-548.

16. Boison D. The adenosine kinase hypothesis of epileptogenesis. Prog Neurobiol 2008;84:249-262.

17. Boison D. Adenosine as a modulator of brain activity. Drug News Persp 2007;20:607-611.

18. Boison D. Adenosine kinase, epilepsy and stroke: mechanisms and therapies. Trends Pharmacol Sci 2006;27:652-658.

19. Boison D. Adenosine and epilepsy: from therapeutic rationale to new therapeutic strategies. Neuroscientist 2005;11:25-36.

20. Wilz A, Pritchard EM, Li T, Lan JQ, Kaplan DL, Boison D. Silk polymer-based adenosine release: Therapeutic potential for epilepsy. Biomaterials 2008;29:3609-3616.

21. Li T, Ren G, Lusardi T, Wilz A, et al. Adenosine kinase is a target for the prediction and prevention of epileptogenesis in mice. J Clin Inv 2008; 118:571-582.

22. Cunha RA. Neuroprotection by adenosine in the brain: from A1 receptor activation to A2A receptor blockade. Purinergic Signaling $2005 ; 1: 111-134$

23. Ribeiro JA. What can adenosine neuromodulation do for neuroprotection? Curr Drug Targets CNS Neurol Disord 2005;4:325329.

24. Ribeiro JA, Sebastiao AM, de Mendonca A. Participation of adenosine receptors in neuroprotection. Drug News Perspect 2003;16: $80-86$.

25. Fredholm BB, Chen JF, Cunha RA, Svenningsson P, Vaugeois JM. Adenosine and brain function. Int Rev Neurobiol 2005;63:191270 .

26. Fredholm BB, Chen JF, Masino SA, Vaugeois JM. Actions of adenosine at its receptors in the CNS: Insights from knockouts and drugs. Annu Rev Pharmacol Toxicol 2005;45:385-412.

27. Fredholm BB, Ijzerman AP, Jacobson KA, Klotz KN, Linden J. International Union of Pharmacology. XXV. Nomenclature and classification of adenosine receptors. Pharmacol Rev 2001;53:527552.

28. Benarroch EE. Adenosine and its receptors: multiple modulatory functions and potential therapeutic targets for neurologic disease. Neurology 2008;70:231-236.

29. Cunha RA. Different cellular sources and different roles of adenosine: $\mathrm{A}(1)$ receptor-mediated inhibition through astrocytic-driven volume transmission and synapse-restricted $\mathrm{A}(2 \mathrm{~A})$ receptor-mediated facilitation of plasticity. Neurochem Int 2008;52:65-72.

30. Fedele DE, Li T, Lan JQ, Fredholm BB, Boison D. Adenosine A1 receptors are crucial in keeping an epileptic focus localized. Exp Neurol 2006;200:184-190.

31. Kochanek PM, Vagni VA, Janesko KL, Washington CB, et al. Adenosine A1 receptor knockout mice develop lethal status epilepticus after experimental traumatic brain injury. J Cereb Blood Flow Metab 2006;26:565-575.

32. Boison D. Adenosine as a neuromodulator in neurological diseases. Curr Opin Pharmacol 2008;8:2-7.

33. Gouder N, Fritschy JM, Boison D. Seizure suppression by adenosine A1 receptor activation in a mouse model of pharmacoresistant epilepsy. Epilepsia 2003;44:877-885.

34. Gouder N, Scheurer L, Fritschy J-M, Boison D. Overexpression of adenosine kinase in epileptic hippocampus contributes to epileptogenesis. J Neurosci 2004;24:692-701.

35. Monopoli A, Conti A, Dionisotti S, Casati C, et al. Pharmacology of the highly selective A1 adenosine receptor agonist 2-chloro-N6cyclopentyladenosine. Arzneimittelforschung 1994;44:1305-1312.

36. Boison D, Scheurer L, Zumsteg V, Rülicke T, et al. Neonatal hepatic steatosis by disruption of the adenosine kinase gene. Proc Natl Acad Sci USA 2002;99:6985-6990.
37. Erion MD, Wiesner JB, Rosengren S, Ugarkar BG, Boyer SH, Tsuchiya M. Therapeutic potential of adenosine kinase inhibitors as analgesic agents. Drug Dev Res 2000;50:S14-06.

38. Huber A, Padrun V, Deglon N, Aebischer P, Mohler H, Boison D. Grafts of adenosine-releasing cells suppress seizures in kindling epilepsy. Proc Natl Acad Sci USA 2001;98:7611-7616.

39. Fedele DE, Koch P, Brüstle O, Scheurer L, et al. Engineering embryonic stem cell derived glia for adenosine delivery. Neurosci Lett 2004;370:160-165.

40. Okabe S, Forsberg-Nilsson K, Spiro AC, Segal M, McKay RD. Development of neuronal precursor cells and functional postmitotic neurons from embryonic stem cells in vitro. Mech Dev 1996; 59:89-102.

41. Ciccarelli R, Di Iorio P, Ballerini P, Ambrosini G, et al. Effects of exogenous ATP and related analogues on the proliferation rate of dissociated primary cultures of rat astrocytes. J Neurosci Res 1994; 39:556-566.

42. Li JY, Christophersen NS, Hall V, Soulet D, Brundin P. Critical issues of clinical human embryonic stem cell therapy for brain repair. Trends Neurosci 2008;31:146-153.

43. Zhang SC, Li XJ, Johnson MA, Pankratz MT. Human embryonic stem cells for brain repair? Philos Trans R Soc Lond B Biol Sci 2008;363:87-99.

44. Thomson JA, Itskovitz-Eldor J, Shapiro SS, Waknitz MA, et al. Embryonic stem cell lines derived from human blastocysts. Science 1998;282:1145-1147.

45. Reubinoff BE, Itsykson P, Turetsky T, Pera MF, et al. Neural progenitors from human embryonic stem cells. Nat Biotechnol 2001;19:1134-1140.

46. Gerrard L, Rodgers L, Cui W. Differentiation of human embryonic stem cells to neural lineages in adherent culture by blocking bone morphogenetic protein signaling. Stem Cells 2005;23:1234-1241.

47. Cohen MA, Itsykson P, Reubinoff BE. Neural differentiation of human ES cells. Curr Protoc Cell Biol 2007; Chapter 23:Unit 23.7.

48. Dottori M, Pera MF. Neural differentiation of human embryonic stem cells. Methods Mol Biol 2008;438:19-30.

49. Roy NS, Cleren C, Singh SK, Yang L, Beal MF, Goldman SA Functional engraftment of human ES cell-derived dopaminergic neurons enriched by coculture with telomerase-immortalized midbrain astrocytes. Nat Med 2006;12:1259-1268.

50. Ben-Hur T, Idelson M, Khaner H, Pera M, et al. Transplantation of human embryonic stem cell-derived neural progenitors improves behavioral deficit in Parkinsonian rats. Stem Cells 2004;22:12461255.

51. Yang D, Zhang ZJ, Oldenburg M, Ayala M, Zhang SC. Human embryonic stem cell-derived dopaminergic neurons reverse functional deficit in parkinsonian rats. Stem Cells 2008;26:55-63.

52. Pankratz MT, Li XJ, Lavaute TM, Lyons EA, Chen X, Zhang SC. Directed neural differentiation of human embryonic stem cells via an obligated primitive anterior stage. Stem Cells 2007;25:15111520.

53. Abdallah BM, Kassem M. Human mesenchymal stem cells: from basic biology to clinical applications. Gene Ther 2008;15:109-116.

54. Onda T, Honmou O, Harada K, Houkin K, Hamada H, Kocsis JD. Therapeutic benefits by human mesenchymal stem cells (hMSCs) and Ang-1 gene-modified hMSCs after cerebral ischemia. J Cereb Blood Flow Metab 2008;28:329-340.

55. Andrews EM, Tsai SY, Johnson SC, Farrer JR, et al. Human adult bone marrow-derived somatic cell therapy results in functional recovery and axonal plasticity following stroke in the rat. Exp Neurol 2008;211:588-592.

56. Mahmood A, Lu D, Qu C, Goussev A, Chopp M. Human marrow stromal cell treatment provides long-lasting benefit after traumatic brain injury in rats. Neurosurgery 2005;57:1026-1031; discussion 1026-1031.

57. Ren G, Li T, Lan JQ, Wilz A, Simon RP, Boison D. Lentiviral RNAi-induced downregulation of adenosine kinase in human mesenchymal stem cell grafts: a novel perspective for seizure control. Exp Neurol 2007;208:26-37.

58. Masino SA, Geiger JD. Are purines mediators of the anticonvulsant/neuroprotective effects of ketogenic diets? Trends Neurosci 2008;31:273-278. 